\title{
$\operatorname{Con} f-950512--265$
}

UCRL-JC-120891

PREPRINT

\section{Electron Acceleration in Relativistic Plasma Waves Generated by a Single Frequency Short-Pulse Laser}

C. A. Coverdale, C. B. Darrow, C. D. Decker, W. B. Mori, K.-C. Tzeng, C. E. Clayton, K. A. Marsh, and C. Joshi

This paper was prepared for submittal to the 1995 IEEE Particle Accelerator Conference

Dallas, Texas

May 1-5, 1995

April 27, 1995

This is a preprint of a paper intended forpublication in a journal or proceedings. Since changes may be made before publication, this preprint is made available with the understanding that it will not be cited or reproduced without the permission of the author.

RECEIVED

JUL 251995

OSTI 


\section{DISCLAIMER}

This document was prepared as an account of work sponsored by an agency of the United States Government. Neither the United States Government nor the University of California nor any of their employees, makes any warranty, express or implied, or assumes any legal liability or responsibility for the accuracy, completeness, or usefulness of any information, apparatus, product, or process disclosed, or represents that its use would not infringe privately owned rights. Reference herein to any specific commercial product, process, or service by trade name, trademark, manufacturer, or other wise, does not necessarily constitute or imply its endorsement, recommendation, or favoring by the United States Government or the University of California. The views and opinions of authors expressed herein do not necessarily state or reflect those of the United States Government or the University of California, and shall not be used for advertising or product endorsement purposes. 


\section{DISCLAIMER}

Portions of this document may be illegible in electronic image products. Images are produced from the best available original document. 


\title{
Electron Acceleration in Relativistic Plasma Waves Generated by a Single Frequency Short-Pulse Laser
}

\author{
C.A. Coverdale, C.B. Darrow, C.D. Decker \\ Lawrence Livermore National Lab, P.O. Box 808, Livermore, CA 94550 \\ W.B. Mori, K.-C. Tzeng, C.E. Clayton, K.A. Marsh, and C. Joshi, \\ Departments of Physics and Electrical Engineering, \\ University of California, Los Angeles, CA 90024
}

\begin{abstract}
Experimental evidence for the acceleration of electrons in a relativistic plasma wave generated by Raman forward scattering (SRS-F) of a single-frequency short pulse laser are presented. A $1.053 \mu \mathrm{m}, 600 \mathrm{fsec}, 5 \mathrm{TW}$ laser was focused into a gas jet with a peak intensity of $8 \times 10^{17}$ W/ $\mathrm{cm}^{2}$. At a plasma density of $2 \times 10^{19} \mathrm{~cm}^{-3}, 2 \mathrm{MeV}$ electrons were detected and their appearance was correlated with the anti-Stokes laser sideband generated by SRS-F. The results are in good agreement with 2-D PIC simulations. The use of short pulse lasers for making ultrahigh gradient accelerators is explored.
\end{abstract}

\section{INTRODUCTION}

There have been many recent analyses on the stability of short laser pulses propagating through underdense plasmas.[1]-[5]. These analyses have shown that such pulses are susceptible to several instabilities which can generate large plasma-wave wakes. One such instability is stimulated Raman forward scattering (SRS-F). SRS-F is a parametric instability in which an incident electromagnetic wave $\left(\omega_{0}, k_{0}\right)$ decays into an electron plasma wave $\left(\omega_{p}, k_{p}\right)$ and two forward propagating electromagnetic waves at frequency $\omega_{0}-\omega_{p}$ (Stokes) and $\omega_{0}+\omega_{p}$ (anti-Stokes), where $\omega_{\mathrm{p}}=\left(4 \pi \mathrm{e}^{2} \mathrm{n}_{\mathrm{o}} / \mathrm{m}\right)^{1 / 2} \cdot[6]$ Energetic electrons are generated by the resulting electron plasma wave. The simultaneous observation of the Stokes and anti-Stokes features and energetic electrons is the strongest evidence for the excitation of SRS-F. The few previous experimental observations of the spectral signature of SRS-F[7] and the energetic electrons associated with the Raman forward scatter[8] were obtained using long pulse lasers ( $\geq 10 \mathrm{psec}$ ). The development of short pulse, high intensity laser systems in recent years has provided a new experimental regime in which to study SRS-F. The experiments and supporting particle-in-cell (PIC) simulations described here show both the spectral features and accelerated electrons, clear evidence of forward stimulated Raman scattering in short pulse, high intensity, underdense plasma interactions.

\section{EXPERIMENTS}

In these experiments, a $1.053 \mu \mathrm{m}, 600 \mathrm{fsec}, 5 \mathrm{TW}$ laser was focused with an $f / 8.2$ aspheric lens into a burst of helium exiting a gas jet, generating a plasma approximately $0.8 \mathrm{~mm}$ long.[9] The peak laser intensity used was approximately $8 \times 10^{17} \mathrm{~W} / \mathrm{cm}^{2}\left(v_{\text {osc }} / \mathrm{c}=0.8\right)$. The plasma density was varied by varying the backing pressure of the gas jet (200-1000 psi); the plasma density ranged from $1 \times 10^{19} \mathrm{~cm}^{-3}$ to $2.5 \times 10^{19} \mathrm{~cm}^{-3}$. Near forward scattered light $\left(5^{\circ}-7^{\circ}\right.$ from the laser axis) was collected and spectrally analyzed using a $0.25 \mathrm{~m}, 150$ grooves $/ \mathrm{mm}$ spectrometer coupled to a liquid nitrogen cooled silicon CCD camera.

The first and second anti-Stokes features were clearly observable in these spectra, as shown in figure 1. The $\Delta \omega$ between the first and second anti-Stokes and between the pump and the first anti-Stokes is the same to within $3 \%$. Poor sensitivity of the CCD for wavelengths greater than $1100 \mathrm{~nm}$ precluded observation of the Stokes features. Although not shown here, the density inferred from the frequency shift of the first anti-Stokes feature monotonically increased with the backing pressure of the gas jet, as expected.

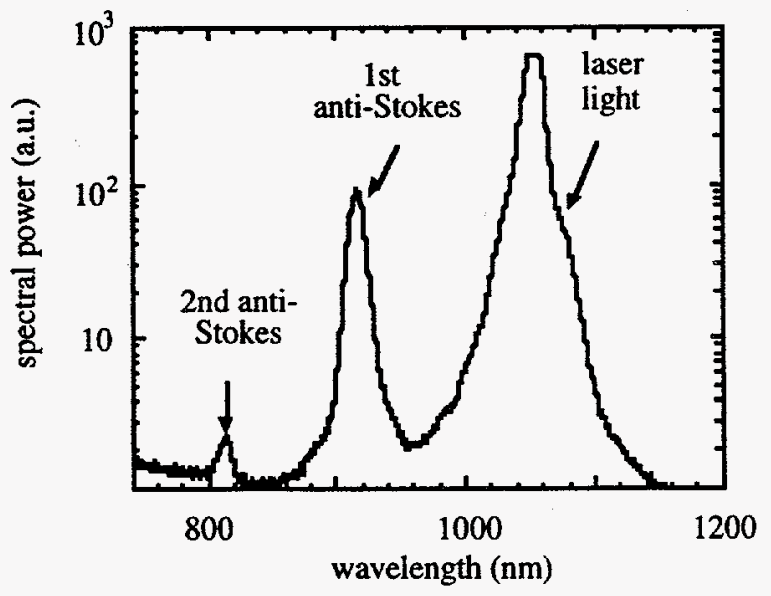

Figure 1: Spectrum of the near-forward scattered light $\left(5^{\circ}-\right.$ $7^{\circ}$ from the laser axis) showing the pump and two antiStokes sidebands. 
High energy electrons produced by the forward SRS were measured with a $90^{\circ}$ bending magnet electron spectrometer placed eight centimeters past the jet in the direct forward path of the laser. Electrons escaping the plasma in an $f / 4$ cone were deflected by the magnet and detected using a $1 \mathrm{~mm}$ thick silicon surface barrier detector (SBD). The magnetic field $(1.5 \mathrm{kG})$ was chosen to collect 2.0 $\pm 0.1 \mathrm{MeV}$ electrons in these experiments. The SBD was shielded to minimize the direct $\mathrm{x}$-ray flux. Additional shielding blocked the line of site between the plasma region and the SBD, ensuring that electrons reaching the SBD were deflected with the magnet: A second SBD was placed $15 \mathrm{~cm}$ from the gas jet $135^{\circ}$ from the laser propagation direction to monitor $x$-ray signal. The $x$-ray contribution to the signal on the electron SBD was measured by reversing the magnet polarity and correlating the $x$-ray signals measured on the two detectors. The electron-plus- $x$-ray was then measured on the electron detector with the correct polarity $B$ field while the control detector measured the $x$-ray contribution. In this manner, the $x$-ray contribution (typically about $10 \%$ ) to the electron signal could be subtracted.

The electron measurements were made at or near 800 psi, the pressure at which the anti-Stokes levels were highest. The electron signal was generally seen to increase with an increase in the anti-Stokes level whereas the $\mathrm{x}$-ray noise level remained more-or-less constant. This correlation is illustrated in figure 2 (open circles). The results of a null experiment are also shown in figure 2 (solid triangles). The null values were obtained by reversing the polarity of the magnet, i.e., deflecting energetic electrons away from the electron SBD.

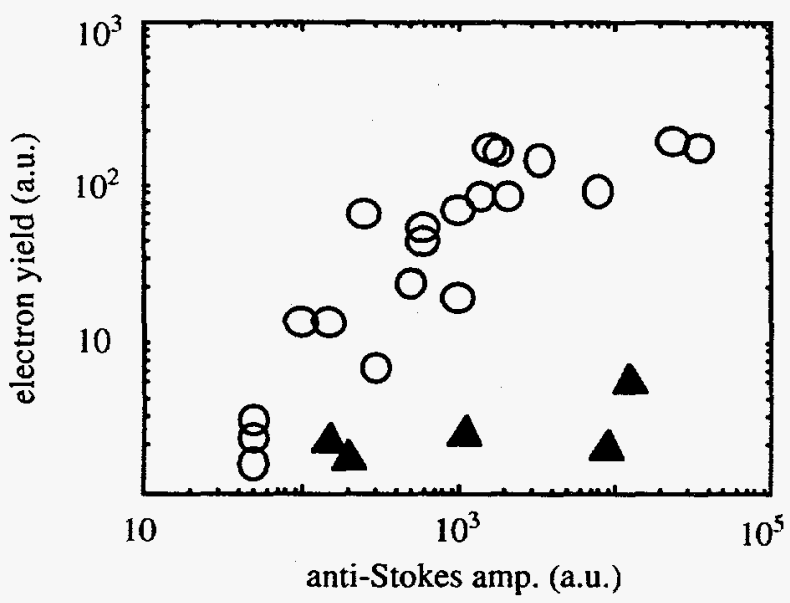

Figure 2: Correlation of the $2.0 \mathrm{MeV}$ electron signal with the amplitude of the first anti-Stokes feature (open circles). The solid triangles represent signal on the electron detector when the magnet polarity was reversed (null tests).

\section{SIMULATIONS}

The advent of massively parallel supercomputers has made possible more realistic modeling of short pulse laserplasma experiments than in the past; i.e., the spatial dimensions (transverse and longitudinal), laser pulsewidth, laser to plasma frequency ratio, and propagation distance in the simulation are the same as in the experiments. We have develped a parallelized fully relativistic, two dimensional electromagnetic PIC code which uses the basic algorithm of ISIS on a cyclic mesh.[5] The computational box is a $8192 \times 256$ cartesian grid and the simulations follow $1.2 \times 10^{7}$ particles for $3 \times 10^{4}$ time steps. The main difficulties with quantitatively modeling the experiment are that the noise level in the simulation is typically larger than than in the experiment and that the simulations are two dimensional (slab geometry).

In order to model the experimental conditions accurately, an estimate of the effective plasma length is required. This estimate was made by performing a series of simulations which covered a range of laser intensities and plasma densities: (A) $\omega_{\mathrm{o}} / \omega_{\mathrm{p}}=10, v_{\mathrm{osd}} \mathrm{c}=0.33$, (B) $\omega_{0} / \omega_{\mathrm{p}}=10, v_{\mathrm{sc}} / \mathrm{c}=0.8$, and $(\mathrm{C}) \omega_{\mathrm{o}} / \omega_{\mathrm{p}}=5, \mathrm{v}_{\mathrm{osc}} / \mathrm{c}=0.5$. In each simulation, the laser pulse duration was $600 \mathrm{fsec}$, the plasma was preformed with a uniform density, and the ions were immobile. The evolution of the electron distribution function and the electromagnetic mode spectra were monitored as a function of distance the rising edge of the laser pulse propagated into the plasma.

In figure 3, we show for simulation $B$ the electron distribution function, $f\left(P_{x}\right)$, and the $k$ spectrum of the laser, $E_{z}\left(k_{x}, k_{y}\right)$, after the pulse has penetrated $0.48 \mathrm{~mm}$ and 0.64 $\mathrm{mm}$ into the plasma. We calculate the total energy within each anti-Stokes line and find that after $0.64 \mathrm{~mm}(0.48$ $\mathrm{mm}$ ), the ratio of anti-Stokes/pump $=0.06(0.006)$ and second anti-Stokes/anti-Stokes $=0.04$ (in the noise). The experimentally measured ratios at $5^{\circ}-7^{\circ}$ for the antiStokes/pump and the 2 nd anti-Stokes/1st anti-Stokes were 0.034 and 0.027 respectively. The second anti-Stokes was not seen on every shot and there was substantial shot-toshot variation (factor of 2) in the normalized anti-Stokes level. The results from the experiment and the simulations are in reasonable agreement, within the shot-to-shot variation, for a plasma length of $0.64 \mathrm{~mm}$. Importantly this distance is less than the presumed dimension of the gas jet. In addition, the edge of the electron distribution does not exceed $2 \mathrm{MeV}$ until the laser has penetrated $0.48 \mathrm{~mm}$ into the plasma (figure 3(a)). It extends to $5 \mathrm{MeV}$ after 0.64 $\mathrm{mm}$ of plasma, with a few electrons at $20 \mathrm{MeV}$ (figure 3(b)). We emphasize that in simulation $A\left(v_{O S d} d c=0.33\right)$, no $\mathrm{MeV}$ electrons or higher order anti-Stokes sidebands were observed for laser penetration distances less than 1 
[5] C. B. Decker, PhD. Dissertation, UCLA-PPG \#1526

$\mathrm{mm}$. Furthermore, the simulations show that if the interaction length is extended to $1.3 \mathrm{~mm}$, the maximum energy of the self-trapped electrons approaches $60 \mathrm{MeV}$.

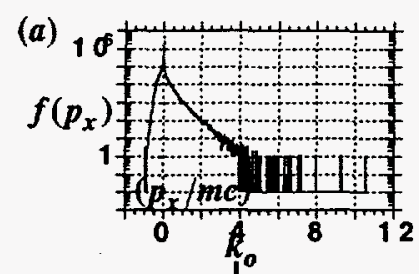

(c)

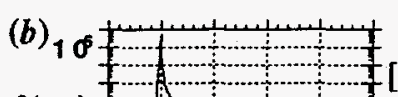

$f\left(p_{x}\right)$

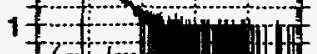

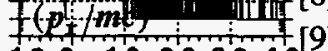
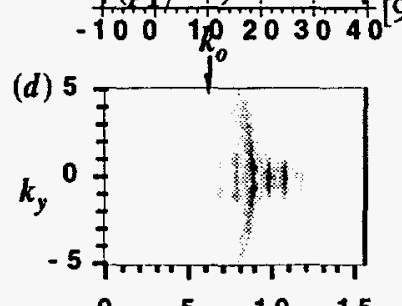
(1994); C. B. Decker et. al., Phys. Rev. E 50, R3338 (1994).

[6] D.W. Forslund et. al., Phys. Fluids 18, 1002 (1975) and references therein; K. Estabrook and W.L. Kruer, Phys. Fluids 26, 1892 (1983).

[7] R.E. Turner et. al., Phys. Rev. Lett. 57, 1725 (1986); S.H. Batha et. al., Phys. Rev. Lett. 66, (1991).

] C. Joshi et. al., Phys. Rev. Lett. 47, 1285 (1981).

C. Clayton and M. Everett, submitted to Rev. Sci. Instr.

Figure 3: The electrotudistribution function at (a) $\$ 48 \mathrm{~mm}$ and (b) $0.64 \mathrm{~mm}$ and the corresponding electromagnetic $k$ spectrum at (c) $0.48 \mathrm{~mm}$ and (d) $0.64 \mathrm{~mm}$ for $\omega_{\mathrm{o}} / \omega_{\mathrm{p}}=10$ and $v_{o s d} / c=0.8$.

\section{CONCLUSIONS}

To summarize, forward stimulated Raman scatter was observed in short pulse, high intensity laser, underdense plasma interaction experiments. Two anti-Stokes features were resolved in the near-forward spectra. Electrons accelerated to $2 \mathrm{MeV}$ were monitored and correlated with the first anti-Stokes feature of the SRS-F. The experimental results are in good agreement with PIC simulations results. The simulations show that if the interaction length could be extended to $1.3 \mathrm{~mm}$, the electrons would be accelerated to energies as high as 60 $\mathrm{MeV}$ by gradients of order $100 \mathrm{GeV} / \mathrm{m}$. Therefore a short pulse, single-frequency laser could form the basis of a robust, ultra-high gradient, modest beam quality electron accelerator.

This work is supported by U.S. DOE grant number DE-FG03-92ER40727 (UCLA) and DOE contract number W-7405-ENG-48 (LLNL).

\section{REFERENCES}

[1] E. Easery et. al., Phys. Rev. Lett., 72, 2887 (1994); J. Krall et. al, Phys. Rev. E 48, 2157 (1993).

[2] T. Antonsen and P. Mora, Phys. Rev. Lett. 69, 2204 (1992); Phys. Flu. B 5, 1440 (1993).

[3] N. E. Adreev et. al., JETP Lett. 55, 571 (1992).

[4] W. B. Mori et. al., Phys. Rev. Lett. 72, 1482 (1994) 